\title{
Near-Integrability of Low-Dimensional Periodic Klein-Gordon Lattices
}

\section{Ognyan Christov (iD}

Faculty of Mathematics and Informatics, Sofia University, 5 J. Bourchier Blvd., 1164 Sofia, Bulgaria

Correspondence should be addressed to Ognyan Christov; christov@fmi.uni-sofia.bg

Received 30 September 2017; Accepted 24 December 2017; Published 22 January 2018

Academic Editor: Alkesh Punjabi

Copyright (C) 2018 Ognyan Christov. This is an open access article distributed under the Creative Commons Attribution License, which permits unrestricted use, distribution, and reproduction in any medium, provided the original work is properly cited.

The low-dimensional periodic Klein-Gordon lattices are studied for integrability. We prove that the periodic lattice with two particles and certain nonlinear potential is nonintegrable. However, in the cases of up to six particles, we prove that their BirkhoffGustavson normal forms are integrable, which allows us to apply KAM theory in most cases.

\section{Introduction}

In this article we deal with the periodic Klein-Gordon (KG) lattice (see, e.g., [1] and references therein) described by the Hamiltonian

$$
\begin{aligned}
& H=\sum_{j \in \mathbb{Z} / n \mathbb{Z}}\left[\frac{p_{j}^{2}}{2}+\frac{C}{2}\left(q_{j+1}-q_{j}\right)^{2}+V\left(q_{j}\right)\right], \\
& p_{j}=\dot{q}_{j}
\end{aligned}
$$

The constant $C>0$ measures the interaction to nearest neighbor particles (with unit masses) and $V(x)$ is a nonlinear potential.

We study the integrability of (1). When $C=0$ the Hamiltonian is separable and, hence, integrable. There exist plenty of periodic or quasi-periodic solutions in the dynamics of (1). It is natural to investigate whether this behavior persists for $C$ small enough (see, e.g., [2]). Here we assume that $C$ is neither very small nor too large.

We are interested in the behavior at low energy; that is why the following main assumptions are in order:

$$
\begin{aligned}
V(x) & =\frac{a}{2} x^{2}+\frac{b}{2} x^{4}, \\
a & >0 \text { irrational. }
\end{aligned}
$$

Remark 1. Such types of potentials are frequently used in the literature [1]. As it can be seen below the choice of $a$ simplifies considerably the calculations.
We can also assume that $C=1$ which can be achieved by rescaling of $t$. Then our Hamiltonian takes the form

$$
\begin{array}{r}
H \\
=\sum_{j \in \mathbb{Z} / n \mathbb{Z}}\left[\frac{p_{j}^{2}}{2}+\frac{1}{2}\left(q_{j+1}-q_{j}\right)^{2}+\frac{a}{2}\left(q_{j}\right)^{2}+\frac{b}{2}\left(q_{j}\right)^{4}\right], \\
p_{j}=\dot{q}_{j} .
\end{array}
$$

Our first result concerns the Hamiltonian with two degrees of freedom $\left(q_{2}=q_{0}\right)$; that is,

$$
\begin{aligned}
H= & \frac{1}{2}\left(p_{1}^{2}+p_{2}^{2}\right)+\frac{1}{2}\left(2 q_{1}^{2}-4 q_{1} q_{2}+2 q_{2}^{2}\right) \\
& +\frac{a}{2}\left(q_{1}^{2}+q_{2}^{2}\right)+\frac{b}{2}\left(q_{1}^{4}+q_{2}^{4}\right) .
\end{aligned}
$$

It simply says that the corresponding Hamiltonian system is integrable only when it is linear.

Theorem 2. The periodic KG lattice with $n=2$ is nonintegrable unless $b=0$.

The above result suggests that generically the periodic KG lattice is nonintegrable for $n \geq 2$. Still there might be some special cases of integrability, while the opposite is not proven rigorously.

Motivated by the works of Rink and Verhulst [3, 4], who presented the periodic FPU chain as a perturbation of an 
integrable and KAM nondegenerated system, namely, the truncated Birkhoff-Gustavson normal form of order 4 in the neighborhood of an equilibrium, our aim is to verify whether this can be done for the low-dimensional KG lattices.

One should note that Rink and Verhulst's result is due to the special symmetry and resonance properties of the FPU chain and should not be expected for lower-order resonant Hamiltonian systems (see, e.g., [5]).

We summarize our second result concerning the integrability of truncated resonant normal forms of the periodic KG lattices up to six particles in the following.

Theorem 3. The truncated normal form $\bar{H}=H_{2}+\bar{H}_{4}$ of the periodic KG lattice is

(i) completely integrable and KAM nondegenerated for $n=2,3,4$;

(ii) completely integrable and KAM nondegenerated when $n=5$ for all $a$ but one;

(iii) completely integrable for $n=6$.

As a consequence from this result, we may conclude for the low-dimensional KG lattices when KAM theory applies that there exist many quasi-periodic solutions of small energy on a long time scale (see Section 2 and for more detailed explanation [3]) and chaotic orbits are of small measure.

The paper is organized as follows. In Section 2 some notions and facts used in the paper are given. We discuss also the appearance of some additional resonant relations. In Section 3 we calculate the Birkhoff-Gustavson normal forms for the cases up to six particles and show that they are integrable in most cases. We finish with some concluding remarks as well as some possible lines of further study.

The proof of Theorem 2 is based on the Ziglin-MoralesRamis theory and since it is more algebraic in nature, it is carried out in the Appendix.

\section{Resonances and Normalization}

In this section we recall briefly some notions and facts about integrability of Hamiltonian systems, action-angle variables, perturbation of integrable systems, and normal forms. More complete exposition can be found in [6-8].

Let $H$ be an analytic Hamiltonian defined on a $2 n$ dimensional symplectic manifold. The corresponding Hamiltonian system is

$$
\dot{x}=X_{H}(x) \text {. }
$$

It is said that a Hamiltonian system is completely integrable if there exist $n$ independent integrals $F_{1}=H, F_{2}, \ldots, F_{n}$ in involution, namely, $\left\{F_{i}, F_{j}\right\}=0$ for all $i$ and $j$, where $\{$,$\} is$ the Poisson bracket. On a neighborhood $U$ of the connected compact level sets of the integrals $M_{c}=\left\{F_{j}=c_{j}, j=\right.$ $1, \ldots, n\}$ by Liouville-Arnold theorem one can introduce a special set of symplectic coordinates, $I_{j}, \varphi_{j}$, called actionangle variables. Then, the integrals $F_{1}=H, F_{2}, \ldots, F_{n}$ are functions of action variables only and the flow of $X_{H}$ is simple

$$
\begin{aligned}
& \dot{I}_{j}=0, \\
& \dot{\varphi}_{j}=\frac{\partial H}{\partial I_{j}}, \\
& \quad j=1, \ldots, n .
\end{aligned}
$$

Therefore, near $M_{c}$, the phase space is foliated with $X_{F_{i}}$ invariant tori over which the flow of $X_{H}$ is quasi-periodic with frequencies $\left(\omega_{1}(I), \ldots, \omega_{n}(I)\right)=\left(\partial H / \partial I_{1}, \ldots, \partial H / \partial I_{n}\right)$.

The map

$$
\left(I_{1}, I_{2}, \ldots, I_{n}\right) \longrightarrow\left(\frac{\partial H}{\partial I_{1}}, \frac{\partial H}{\partial I_{2}}, \ldots, \frac{\partial H}{\partial I_{n}}\right)
$$

is called frequency map.

Consider a small perturbation of an integrable Hamiltonian $H_{0}$. According to Poincaré the main problem of mechanics is to study the perturbation of quasi-periodic motions in the system given by the Hamiltonian

$$
H=H_{0}(I)+\varepsilon H_{1}(I, \varphi), \quad \varepsilon \ll 1 .
$$

KAM theory [9-11] gives conditions on the integrable Hamiltonian $H_{0}$ which ensures the survival of the most of the invariant tori. The following condition, usually called Kolmogorov's condition, is that the frequency map should be a local diffeomorphism, or equivalently

$$
\operatorname{det}\left(\frac{\partial^{2} H_{0}}{\partial I_{i} \partial I_{j}}\right) \neq 0
$$

on an open and dense subset of $U$. We should note that the measure of the surviving tori decreases with the increase of both perturbation and the measure of the set where above Hessian is too close to zero.

In a neighborhood of the equilibrium $(q, p)=(0,0)$ we have the following expansion of $H$ :

$$
\begin{aligned}
H & =H_{2}+H_{3}+H_{4}+\cdots, \\
H_{2} & =\sum \omega_{j}\left(q_{j}^{2}+p_{j}^{2}\right), \quad \omega_{j}>0 .
\end{aligned}
$$

We assume that $H_{2}$ is a positively defined quadratic form. The frequency $\omega=\left(\omega_{1}, \ldots, \omega_{n}\right)$ is said to be in resonance if there exists a vector $k=\left(k_{1}, \ldots, k_{n}\right), k_{j} \in \mathbb{Z}, j=1, \ldots, n$, such that $(\omega, k)=\sum k_{j} \omega_{j}=0$, where $|k|=\sum\left|k_{j}\right|$ is the order of resonance.

With the help of a series of canonical transformations close to the identity, $H$ simplifies. In the absence of resonances the simplified Hamiltonian is called Birkhoff normal form and, otherwise, Birkhoff-Gustavson normal form, which may contain combinations of angles arising from resonances.

Often to detect the behavior in a small neighborhood of the equilibrium, instead of the Hamiltonian $H$ one considers the normal form truncated to some order

$$
\bar{H}=H_{2}+\cdots+\bar{H}_{m} .
$$


TABLE 1

\begin{tabular}{cc}
\hline$n$ & $\Omega_{k}$ \\
\hline 2 & $\sqrt{a+4}, \sqrt{a}$ \\
4 & $\sqrt{a+3}, \sqrt{a+3}, \sqrt{a}$ \\
5 & $\sqrt{a+2}, \sqrt{a+4}, \sqrt{a+2}, \sqrt{a}$ \\
6 & $\sqrt{a+(5-\sqrt{5}) / 2}, \sqrt{a+(5+\sqrt{5}) / 2}, \sqrt{a+(5+\sqrt{5}) / 2}, \sqrt{a+(5-\sqrt{5}) / 2}, \sqrt{a}$ \\
\hline
\end{tabular}

It is known that the truncated to any order Birkhoff normal form is integrable [8]. The truncated Birkhoff-Gustavson normal form has at least two integrals $-H_{2}$ and $\bar{H}$. Therefore, the truncated normal form of two-degree-of-freedom Hamiltonian is integrable.

In order to obtain estimates of the approximation by normalization in a neighborhood of an equilibrium point we scale $q \rightarrow \varepsilon \widetilde{q}, p \rightarrow \varepsilon \widetilde{p}$. Here $\varepsilon$ is a small positive parameter and $\varepsilon^{2}$ is a measure for the energy relative to the equilibrium energy. Then, dividing by $\varepsilon^{2}$ and removing tildes we get

$$
\bar{H}=H_{2}+\varepsilon \bar{H}_{3}+\cdots+\varepsilon^{m-2} \bar{H}_{m} .
$$

Provided that $\omega_{j}>0$ it is proven in [12] that $\bar{H}$ is an integral for the original system with error $O\left(\varepsilon^{m-1}\right)$ and $H_{2}$ is an integral for the original system with error $O(\varepsilon)$ for the whole time interval. If we have more independent integrals, then they are integrals for the original Hamiltonian system with error $O\left(\varepsilon^{m-2}\right)$ on the time scale $1 / \varepsilon$.

The first integrals for the normal form $\bar{H}$ are approximate integrals for the original system; that is, if the normal form is integrable, then the original system is near integrable in the above sense.

Returning to the Hamiltonian of the periodic KG lattice (4) we see that its quadratic part $\mathrm{H}_{2}$ is not in diagonal form:

$$
H_{2}=\frac{1}{2} p^{T} p+\frac{1}{2} q^{T} L_{n} q
$$

Here $L_{n}$ is the following $n \times n$ matrix:

$$
L_{n}:=\left(\begin{array}{ccccc}
2+a & -1 & & & -1 \\
-1 & 2+a & -1 & & \\
& \ddots & \ddots & \ddots & \\
& & & & \\
& & -1 & 2+a & -1 \\
-1 & & & -1 & 2+a
\end{array}\right) .
$$

The eigenvalues of $L_{n}$ are of the form $\Omega_{k}^{2}=a+\omega_{k}^{2}, \omega_{k}=$ $2 \sin (k \pi / n)$. In order to obtain the corresponding eigenvectors $y^{k}$, following [3] we define

$$
y^{n}:=\frac{1}{\sqrt{n}}(1,1, \ldots, 1)^{T}
$$

and if $n$ is even,

$$
y^{n / 2}:=\frac{1}{\sqrt{n}}(1,-1,1,-1, \ldots,-1)^{T} .
$$

Further, for $1 \leq k<n / 2$, we define $y^{k}$ and $y^{n-k}$ via their coordinates

$$
\begin{aligned}
y_{j}^{k} & :=\sqrt{\frac{2}{n}} \cos \left(\frac{2 k j \pi}{n}\right), \\
y_{j}^{n-k} & :=\sqrt{\frac{2}{n}} \sin \left(\frac{2 k j \pi}{n}\right) .
\end{aligned}
$$

It is easily checked that $\left\{y^{1}, \ldots, y^{n}\right\}$ is an orthonormal basis of $\mathbb{R}^{n}$, consisting of eigenvectors of $L_{n}$. Let $Y$ be the $n \times n$ matrix formed by the vectors $y^{k}$ as columns; then $Y^{T} Y=$ Id, $Y^{-1} L_{n} Y=\Omega:=\operatorname{diag}\left(\Omega_{1}^{2}, \ldots, \Omega_{n}^{2}\right)$. The symplectic Fourier-transformation $q=Y \bar{q}, p=Y \bar{p}$ brings $H_{2}$ in diagonal form

$$
H_{2}=\frac{1}{2} p^{T} p+\frac{1}{2} q^{T} \Omega q .
$$

The variables $(\bar{q}, \bar{p})$ are known as phonons.

We need one more definition.

Definition 4 (see [3]). It is said that $\omega \in \mathbb{R}^{n}$ satisfies the property of internal resonance if for any $k \in \mathbb{Z}^{n}$ with $(k, \omega)=$ $0,|k|=4$, we have $k_{j}=-k_{n-j}$ when $1 \leq j<n / 2$.

In Table 1 we list the frequencies $\Omega_{k}$ of some lowdimensional periodic KG lattice.

As it is seen from the table we almost always have internal resonances. In general, $\Omega_{k}=\Omega_{j}$ holds if only if $j=n-k$. There are plenty of resonances when $a \in \mathbb{Q}$.

Nevertheless, the assumption on $a$ (3) does not prevent the appearance of more complicated resonances. Indeed, in the case of 4 particles there exists unique $a>0$ irrational, for which the following resonant relations are fulfilled $\left(\Omega_{1}=\right.$ $\left.\Omega_{3}\right)$ :

$$
\begin{aligned}
& \Omega_{2}-\Omega_{3}-\Omega_{4}=0 \\
& \Omega_{2}-\Omega_{1}-\Omega_{4}=0 .
\end{aligned}
$$

There are many like them and in particular for $n=5$ and $n=6$. Luckily, these resonances are of no importance for our considerations, because they produce third-order resonant terms and none of them appears due to the assumption on $V(x)(2)$. 
The resonances we have to worry about are the forthorder ones. Trivial inspection shows that for $a$ in the domain (3), there are no such resonances when $n=2,3,4,6$. However, there are some of that kind when $n=5\left(\Omega_{1}=\Omega_{4}, \Omega_{2}=\Omega_{3}\right)$; namely,

(1) for $a_{1}=(5-\sqrt{5}) / 16$ the following resonant relations are fulfilled:

$$
\begin{aligned}
& \Omega_{1}-3 \Omega_{5}=0 \\
& \Omega_{4}-3 \Omega_{5}=0
\end{aligned}
$$

(2) for $a_{2}=(5+\sqrt{5}) / 16$ the following resonant relations are fulfilled:

$$
\begin{aligned}
& \Omega_{2}-3 \Omega_{5}=0, \\
& \Omega_{3}-3 \Omega_{5}=0,
\end{aligned}
$$

(3) for $a_{3}=5(3 \sqrt{5}-5) / 16$ the following resonant relations are fulfilled:

$$
\begin{aligned}
& 2 \Omega_{1}-\Omega_{2}-\Omega_{5}=0, \\
& 2 \Omega_{4}-\Omega_{2}-\Omega_{5}=0, \\
& 2 \Omega_{1}-\Omega_{3}-\Omega_{5}=0, \\
& 2 \Omega_{4}-\Omega_{3}-\Omega_{5}=0 .
\end{aligned}
$$

We will see how these additional resonances affect $\bar{H}_{4}$ in the end of Section 3.4.

\section{Low-Dimensional Lattices}

In this section we calculate the normal forms for the periodic KG lattices with particles up to six and in this way we prove Theorem 3. We do not use $\varepsilon$ in the fourth-degree expression, but keep in mind that we are close to the equilibrium. Of course, it is assumed that $b \neq 0$.

3.1. Two Particles. This case is easy. It is well known that the truncated to any order normal form of a two-degree-offreedom Hamiltonian is integrable. It remains only to verify the KAM condition.

We have already brought the quadratic part of the Hamiltonian in diagonal form. It is important that it is written in the phonons $(\bar{q}, \bar{p})$.

$$
\begin{aligned}
H= & \frac{1}{2}\left(\bar{p}_{1}^{2}+\bar{p}_{2}^{2}\right)+\frac{1}{2}\left[(4+a) \bar{q}_{1}^{2}+a \bar{q}_{2}^{2}\right] \\
& +\frac{b}{4}\left(\bar{q}_{1}^{4}+6 \bar{q}_{1}^{2} \bar{q}_{2}^{2}+\bar{q}_{2}^{4}\right) .
\end{aligned}
$$

Further, we perform a scaling

$$
\begin{aligned}
& \bar{q}_{1} \longrightarrow \frac{1}{\sqrt[4]{a+4}} \bar{q}_{1}, \\
& \bar{p}_{1} \longrightarrow \sqrt[4]{a+4} \bar{p}_{1}
\end{aligned}
$$

$$
\begin{aligned}
& \bar{q}_{2} \longrightarrow \frac{1}{\sqrt[4]{a}} \bar{q}_{2}, \\
& \bar{p}_{2} \longrightarrow \sqrt[4]{a} \bar{p}_{2},
\end{aligned}
$$

which preserves the symplectic form. The Hamiltonian (25) becomes

$$
\begin{aligned}
H= & \frac{\sqrt{a+4}}{2}\left(\bar{p}_{1}^{2}+\bar{q}_{1}^{2}\right)+\frac{\sqrt{a}}{2}\left(\bar{p}_{2}^{2}+\bar{q}_{2}^{2}\right) \\
& +\frac{b}{4}\left(\frac{\bar{q}_{1}^{4}}{a+4}+\frac{6 \bar{q}_{1}^{2} \bar{q}_{2}^{2}}{\sqrt{a(a+4)}}+\frac{\bar{q}_{2}^{4}}{a}\right) .
\end{aligned}
$$

Usually at this place one makes the following change of variables:

$$
\begin{aligned}
& \bar{q}_{j}=\frac{1}{2}\left(z_{j}+w_{j}\right), \\
& \bar{p}_{j}=\frac{1}{2 i}\left(z_{j}-w_{j}\right), \\
& \quad j=1,2 .
\end{aligned}
$$

Since the frequencies $\Omega_{1}=\sqrt{a+4}, \Omega_{2}=\sqrt{a}$ are incommensurable, the only resonant terms which remain are

$$
z_{j} w_{j},\left(z_{j} w_{j}\right)^{2}, j=1,2, z_{1} w_{1} z_{2} w_{2} \text {. }
$$

The other terms can be removed via symplectic near-identity change. Therefore, the normal form of (25) up to order four $\bar{H}=H_{2}+\bar{H}_{4}$ is

$$
\begin{aligned}
\bar{H}= & \frac{\sqrt{a+4}}{2} z_{1} w_{1}+\frac{\sqrt{a}}{2} z_{2} w_{2} \\
& +\frac{3 b}{32}\left[\frac{\left(z_{1} w_{1}\right)^{2}}{a+4}+4 \frac{z_{1} w_{1} z_{2} w_{2}}{\sqrt{a(a+4)}}+\frac{\left(z_{2} w_{2}\right)^{2}}{a}\right]
\end{aligned}
$$

or, returning to the $(\bar{q}, \bar{p})$ coordinates, we get

$$
\begin{aligned}
\bar{H} & =\frac{\sqrt{a+4}}{2}\left(\bar{p}_{1}^{2}+\bar{q}_{1}^{2}\right)+\frac{\sqrt{a}}{2}\left(\bar{p}_{2}^{2}+\bar{q}_{2}^{2}\right) \\
& +\frac{3 b}{32}\left[\frac{\left(\bar{p}_{1}^{2}+\bar{q}_{1}^{2}\right)^{2}}{a+4}+4 \frac{\left(\bar{p}_{1}^{2}+\bar{q}_{1}^{2}\right)\left(\bar{p}_{2}^{2}+\bar{q}_{2}^{2}\right)}{\sqrt{a(a+4)}}\right. \\
& \left.+\frac{\left(\bar{p}_{2}^{2}+\bar{q}_{2}^{2}\right)^{2}}{a}\right] .
\end{aligned}
$$

This normal form is clearly integrable with quadratic first integrals $I_{j}=\bar{p}_{j}^{2}+\bar{q}_{j}^{2}, \quad j=1,2$.

Finally, introducing symplectic polar coordinates, which are action-angle variables

$$
\begin{aligned}
& \bar{q}_{j}=\sqrt{2 I_{j}} \cos \varphi_{j}, \\
& \bar{p}_{j}=\sqrt{2 I_{j}} \sin \varphi_{j}, \\
& j=1,2
\end{aligned}
$$


we get

$$
\begin{aligned}
\bar{H}= & \sqrt{a+4} I_{1}+\sqrt{a} I_{2} \\
& +\frac{3 b}{8}\left[\frac{I_{1}^{2}}{a+4}+4 \frac{I_{1} I_{2}}{\sqrt{a(a+4)}}+\frac{I_{2}^{2}}{a}\right] .
\end{aligned}
$$

It can easily be checked that Kolmogorov's condition is valid.

3.2. Three Particles. First, we make use of the phonons $(\bar{q}, \bar{p})$ (as explained in Section 2) to transform the quadratic part of the Hamiltonian (4) $n=3$ in diagonal form

$$
\begin{aligned}
H & =\frac{1}{2}\left(\bar{p}_{1}^{2}+\bar{p}_{2}^{2}+\bar{p}_{3}^{2}\right)+\frac{a+3}{2}\left(\bar{q}_{1}^{2}+\bar{q}_{2}^{2}\right)+\frac{a}{2} \bar{q}_{3}^{2} \\
& +\frac{b}{18}\left[\frac{9}{2}\left(\bar{q}_{1}^{4}+\bar{q}_{2}^{4}\right)+3 \bar{q}_{3}^{4}+6 \sqrt{2} \bar{q}_{1}^{3} \bar{q}_{3}+9 \bar{q}_{1}^{2} \bar{q}_{2}^{2}\right. \\
& \left.+18\left(\bar{q}_{1}^{2}+\bar{q}_{2}^{2}\right) \bar{q}_{3}^{2}-18 \sqrt{2} \bar{q}_{1} \bar{q}_{2}^{2} \bar{q}_{3}\right] .
\end{aligned}
$$

Further, the scaling

$$
\begin{gathered}
\bar{q}_{1,2} \longrightarrow \frac{1}{\sqrt[4]{a+3}} \bar{q}_{1,2}, \\
\bar{p}_{1,2} \longrightarrow \sqrt[4]{a+3} \bar{p}_{1,2}, \\
\bar{q}_{3} \longrightarrow \frac{1}{\sqrt[4]{a}} \bar{q}_{3}, \\
\bar{p}_{3} \longrightarrow \sqrt[4]{a} \bar{p}_{3}
\end{gathered}
$$

results in

$$
\begin{aligned}
H & =\frac{\sqrt{a+3}}{2}\left(\bar{p}_{1}^{2}+\bar{q}_{1}^{2}+\bar{p}_{2}^{2}+\bar{q}_{2}^{2}\right)+\frac{\sqrt{a}}{2}\left(\bar{p}_{3}^{2}+\bar{q}_{3}^{2}\right) \\
& +\frac{b}{18}\left[\frac{9\left(\bar{q}_{1}^{4}+\bar{q}_{2}^{4}\right)}{2(a+3)}+\frac{3 \bar{q}_{3}^{4}}{a}+\frac{6 \sqrt{2} \bar{q}_{1}^{3} \bar{q}_{3}}{\sqrt[4]{a(a+3)^{3}}}+\frac{9 \bar{q}_{1}^{2} \bar{q}_{2}^{2}}{a+3}\right. \\
& \left.+\frac{18\left(\bar{q}_{1}^{2}+\bar{q}_{2}^{2}\right) \bar{q}_{3}^{2}}{\sqrt{a(a+3)}}-\frac{18 \sqrt{2} \bar{q}_{1} \bar{q}_{2}^{2} \bar{q}_{3}}{\sqrt[4]{a(a+3)^{3}}}\right] .
\end{aligned}
$$

Passing to the variables $\left(z_{j}, w_{j}\right), j=1,2,3$ (28), we notice that there is an internal resonance between the frequencies $\Omega_{1}$ and $\Omega_{2}$. Therefore, the generators of the normal form are

$$
z_{j} w_{j}, j=1,2,3 \text {, and } z_{1} w_{2}, z_{2} w_{1} .
$$

After removing the nonresonant terms, the normal form of the (33) up to order four $\bar{H}=H_{2}+\bar{H}_{4}$ is

$$
\begin{aligned}
\bar{H} & =\frac{\sqrt{a+3}}{2}\left(z_{1} w_{1}+z_{2} w_{2}\right)+\frac{\sqrt{a}}{2} z_{3} w_{3} \\
& +\frac{b}{18}\left[\frac{27\left(z_{1} w_{1}+z_{2} w_{2}\right)^{2}}{16(a+3)}+\frac{9\left(z_{3} w_{3}\right)^{2}}{8 a}\right. \\
& \left.+\frac{9\left(z_{1} w_{1}+z_{2} w_{2}\right) z_{3} w_{3}}{2 \sqrt{a(a+3)}}+\frac{9\left(z_{1} w_{2}-z_{2} w_{1}\right)^{2}}{16(a+3)}\right],
\end{aligned}
$$

or, returning to $(\bar{q}, \bar{p})$, we get

$$
\begin{aligned}
\bar{H} & =\frac{\sqrt{a+3}}{2}\left(\bar{p}_{1}^{2}+\bar{q}_{1}^{2}+\bar{p}_{2}^{2}+\bar{q}_{2}^{2}\right)+\frac{\sqrt{a}}{2}\left(\bar{p}_{3}^{2}+\bar{q}_{3}^{2}\right) \\
& +\frac{b}{2}\left[\frac{3\left(\bar{p}_{1}^{2}+\bar{q}_{1}^{2}+\bar{p}_{2}^{2}+\bar{q}_{2}^{2}\right)^{2}}{16(a+3)}+\frac{\left(\bar{p}_{3}^{2}+\bar{q}_{3}^{2}\right)^{2}}{8 a}\right. \\
& +\frac{\left(\bar{p}_{1}^{2}+\bar{q}_{1}^{2}+\bar{p}_{2}^{2}+\bar{q}_{2}^{2}\right)\left(\bar{p}_{3}^{2}+\bar{q}_{3}^{2}\right)}{2 \sqrt{a(a+3)}} \\
& \left.-\frac{\left(\bar{p}_{1} \bar{q}_{2}-\bar{q}_{1} \bar{p}_{2}\right)^{2}}{4(a+3)}\right] .
\end{aligned}
$$

This normal form is integrable with the following quadratic first integrals:

$$
\begin{aligned}
& F_{1}:=\bar{p}_{1}^{2}+\bar{q}_{1}^{2}+\bar{p}_{2}^{2}+\bar{q}_{2}^{2}, \\
& G_{1}:=\bar{p}_{1} \bar{q}_{2}-\bar{q}_{1} \bar{p}_{2}, \\
& I_{3}:=\bar{p}_{3}^{2}+\bar{q}_{3}^{2} .
\end{aligned}
$$

In order to introduce action-angle variables, we need to find the set of regular values of the energy momentum map

$$
\mathrm{EM}:(\bar{q}, \bar{p}) \longrightarrow\left(F_{1}, G_{1}, I_{3}\right) .
$$

In fact, this is done in [4]. Denote by $U_{r}=\left\{\left(F_{1}, G_{1}, I_{3}\right) \in\right.$ $\left.\mathbb{R}^{3}, F_{1}>0,\left|G_{1}\right|<F_{1}, I_{3}>0\right\}$. Then for all $\left(F_{1}, G_{1}, I_{3}\right) \in U_{r}$ the level sets of $\mathrm{EM}^{-1}\left(F_{1}, G_{1}, I_{3}\right)$ are diffeomorphic to 3-tori.

Let arg : $\mathbb{R}^{2} \backslash\{(0,0)\} \rightarrow \mathbb{R} / 2 \pi \mathbb{Z}$ be the argument function $\arg (r \cos \Phi, r \sin \Phi) \rightarrow \Phi$. Define the following set of variables $\left(F_{1}, G_{1}, I_{3}, \phi_{1}, \psi_{1}, \varphi_{3}\right) F_{1}, G_{1}, I_{3}$ as above and

$$
\begin{aligned}
\phi_{1}:= & \frac{1}{2} \arg \left(-\bar{p}_{2}-\bar{q}_{1}, \bar{p}_{1}-\bar{q}_{2}\right) \\
& +\frac{1}{2} \arg \left(\bar{p}_{2}-\bar{q}_{1}, \bar{p}_{1}+\bar{q}_{2}\right), \\
\psi_{1}:= & \frac{1}{2} \arg \left(-\bar{p}_{2}-\bar{q}_{1}, \bar{p}_{1}-\bar{q}_{2}\right) \\
& -\frac{1}{2} \arg \left(\bar{p}_{2}-\bar{q}_{1}, \bar{p}_{1}+\bar{q}_{2}\right), \\
\varphi_{3}:= & \arctan \frac{\bar{p}_{3}}{\bar{q}_{3}} .
\end{aligned}
$$

Using the formula $d \arg (x, y)=(x d y-y d x) /\left(x^{2}+y^{2}\right)$, one can verify that $\left(F_{1}, G_{1}, I_{3}, \phi_{1}, \psi_{1}, \varphi_{3}\right)$ are indeed canonical coordinates $\sum d \bar{p}_{j} \wedge \bar{q}_{j}=d F_{1} \wedge d \phi_{1}+d G_{1} \wedge d \psi_{1}+d I_{3} \wedge d \varphi_{3}$.

Then the truncated up to order 4 normal form as a function of actions is

$$
\begin{aligned}
\bar{H} & =\frac{\sqrt{a+3}}{2} F_{1}+\frac{\sqrt{a}}{2} I_{3}+\frac{b}{2}\left[\frac{3 F_{1}^{2}}{16(a+3)}\right. \\
& \left.+\frac{F_{1} I_{3}}{2 \sqrt{a(a+3)}}-\frac{G_{1}^{2}}{4(a+3)}+\frac{I_{3}^{2}}{8 a}\right] .
\end{aligned}
$$


Then one can easily check up that Kolmogorov's condition is valid.

Remark 5. If we set $a=1$, we get

$$
\begin{aligned}
\bar{H} & =\frac{2}{2}\left(\bar{p}_{1}^{2}+\bar{q}_{1}^{2}+\bar{p}_{2}^{2}+\bar{q}_{2}^{2}\right)+\frac{1}{2}\left(\bar{p}_{3}^{2}+\bar{q}_{3}^{2}\right) \\
& +\frac{b}{2}\left[\frac{3\left(\bar{p}_{1}^{2}+\bar{q}_{1}^{2}+\bar{p}_{2}^{2}+\bar{q}_{2}^{2}\right)^{2}}{64}+\frac{\left(\bar{p}_{3}^{2}+\bar{q}_{3}^{2}\right)^{2}}{8}\right. \\
& +\frac{\left(\bar{p}_{1}^{2}+\bar{q}_{1}^{2}+\bar{p}_{2}^{2}+\bar{q}_{2}^{2}\right)\left(\bar{p}_{3}^{2}+\bar{q}_{3}^{2}\right)}{4} \\
& \left.-\frac{\left(\bar{p}_{1} \bar{q}_{2}-\bar{q}_{1} \bar{p}_{2}\right)^{2}}{16}\right],
\end{aligned}
$$

which is an example of an integrable KAM nondegenerate normal form of $2: 2: 1$ (or $1: 2: 2$ ) Hamiltonian resonance (see, e.g., [13]).

3.3. Four Particles. It turns out that the normal form of the periodic KG lattice in the case of four particles is surprisingly simple, no matter the internal resonance.

After transforming the quadratic part in diagonal form and scaling

$$
\begin{gathered}
\bar{q}_{1,3} \rightarrow \frac{1}{\sqrt[4]{a+2}} \bar{q}_{1,3}, \\
\bar{p}_{1,3} \longrightarrow \sqrt[4]{a+2} \bar{p}_{1,3}, \\
\bar{q}_{2} \longrightarrow \frac{1}{\sqrt[4]{a+4}} \bar{q}_{2}, \\
\bar{p}_{2} \longrightarrow \sqrt[4]{a+4} \bar{p}_{2}, \\
\bar{q}_{4} \longrightarrow \frac{1}{\sqrt[4]{a}} \bar{q}_{4}, \\
\bar{p}_{4} \longrightarrow \sqrt[4]{a} \bar{p}_{4}
\end{gathered}
$$

the Hamiltonian (4) $n=4$ takes the form

$$
\begin{aligned}
H & =\frac{\sqrt{a+2}}{2}\left(\bar{p}_{1}^{2}+\bar{q}_{1}^{2}\right)+\frac{\sqrt{a+4}}{2}\left(\bar{p}_{2}^{2}+\bar{q}_{2}^{2}\right) \\
& +\frac{\sqrt{a+2}}{2}\left(\bar{p}_{3}^{2}+\bar{q}_{3}^{2}\right)+\frac{\sqrt{a}}{2}\left(\bar{p}_{4}^{2}+\bar{q}_{4}^{2}\right) \\
& +\frac{b}{8}\left[\frac{2\left(\bar{q}_{1}^{4}+\bar{q}_{3}^{4}\right)}{(a+2)}+\frac{\bar{q}_{2}^{4}}{a+4}+\frac{\bar{q}_{4}^{4}}{a}\right. \\
& +\frac{12 \bar{q}_{2} \bar{q}_{3}^{2} \bar{q}_{4}}{\sqrt[4]{a(a+4)(a+2)^{2}}}-\frac{12 \bar{q}_{1}^{2} \bar{q}_{2} \bar{q}_{4}}{\sqrt[4]{a(a+4)(a+2)^{2}}}
\end{aligned}
$$

$$
\begin{aligned}
& +\frac{6 \bar{q}_{2}^{2}\left(\bar{q}_{1}^{2}+\bar{q}_{3}^{2}\right)}{\sqrt{(a+2)(a+4)}}+\frac{6 \bar{q}_{4}^{2}\left(\bar{q}_{1}^{2}+\bar{q}_{3}^{2}\right)}{\sqrt{a(a+2)}} \\
& \left.+\frac{6 \bar{q}_{2}^{2} \bar{q}_{4}^{2}}{\sqrt{a(a+4)}}\right] .
\end{aligned}
$$

There is an internal resonance between the frequencies $\Omega_{1}$ and $\Omega_{3}$. In variables $\left(z_{j}, w_{j}\right), j=1,2,3,4$, the generators of the normal form are

$$
z_{j} w_{j} \text {, and } z_{1} w_{3}, z_{3} w_{1}
$$

However, as it is seen from (44), the variables $\bar{q}_{1}$ and $\bar{q}_{3}$ do not couple, so the last two generators do not appear in the normal form as if there are no resonances. After removing the nonresonant terms, the normal form of (44) up to order four $\bar{H}=H_{2}+\bar{H}_{4}$ reads

$$
\begin{aligned}
\bar{H} & =\frac{\sqrt{a+2}}{2} z_{1} w_{1}+\frac{\sqrt{a+4}}{2} z_{2} w_{2}+\frac{\sqrt{a+2}}{2} z_{3} w_{3} \\
& +\frac{\sqrt{a}}{2} z_{4} w_{4}+\frac{b}{8}\left[\frac{3\left(z_{1} w_{1}\right)^{2}}{4(a+2)}+\frac{3\left(z_{2} w_{2}\right)^{2}}{8(a+4)}\right. \\
& +\frac{3\left(z_{3} w_{3}\right)^{2}}{4(a+2)}+\frac{3\left(z_{4} w_{4}\right)^{2}}{8 a}+\frac{3 z_{2} w_{2}\left(z_{1} w_{1}+z_{3} w_{3}\right)}{\sqrt{2(a+2)(a+4)}} \\
& \left.+\frac{3 z_{4} w_{4}\left(z_{1} w_{1}+z_{3} w_{3}\right)}{\sqrt{2 a(a+2)}}+\frac{3 z_{2} w_{2} z_{4} w_{4}}{\sqrt{2 a(a+4)}}\right]
\end{aligned}
$$

or, in $(\bar{q}, \bar{p})$ variables,

$$
\begin{aligned}
\bar{H} & =\frac{\sqrt{a+2}}{2}\left(\bar{p}_{1}^{2}+\bar{q}_{1}^{2}\right)+\frac{\sqrt{a+4}}{2}\left(\bar{p}_{2}^{2}+\bar{q}_{2}^{2}\right) \\
& +\frac{\sqrt{a+2}}{2}\left(\bar{p}_{3}^{2}+\bar{q}_{3}^{2}\right)+\frac{\sqrt{a}}{2}\left(\bar{p}_{4}^{2}+\bar{q}_{4}^{2}\right) \\
& +\frac{b}{8}\left[\frac{3\left(\bar{p}_{1}^{2}+\bar{q}_{1}^{2}\right)^{2}}{4(a+2)}+\frac{3\left(\bar{p}_{2}^{2}+\bar{q}_{2}^{2}\right)^{2}}{8(a+4)}+\frac{3\left(\bar{p}_{3}^{2}+\bar{q}_{3}^{2}\right)^{2}}{4(a+2)}\right. \\
& +\frac{3\left(\bar{p}_{4}^{2}+\bar{q}_{4}^{2}\right)^{2}}{8 a}+\frac{3\left(\bar{p}_{2}^{2}+\bar{q}_{2}^{2}\right)\left(\bar{p}_{1}^{2}+\bar{q}_{1}^{2}+\bar{p}_{3}^{2}+\bar{q}_{3}^{2}\right)}{\sqrt{2(a+2)(a+4)}} \\
& +\frac{3\left(\bar{p}_{4}^{2}+\bar{q}_{4}^{2}\right)\left(\bar{p}_{1}^{2}+\bar{q}_{1}^{2}+\bar{p}_{3}^{2}+\bar{q}_{3}^{2}\right)}{\sqrt{2 a(a+2)}} \\
& +\frac{3\left(\bar{p}_{2}^{2}+\bar{q}_{2}^{2}\right)\left(\bar{p}_{4}^{2}+\bar{q}_{4}^{2}\right)}{\sqrt{2 a(a+4)}]} .
\end{aligned}
$$

This normal form is integrable with integrals $I_{j}=\bar{p}_{j}^{2}+$ $\bar{q}_{j}^{2}, j=1,2,3,4$ and the action-angle variables are clear. The 
truncated normal form up to order 4 as a function of the action variables is

$$
\begin{aligned}
\bar{H} & =\frac{\sqrt{a+2}}{2} I_{1}+\frac{\sqrt{a+4}}{2} I_{2}+\frac{\sqrt{a+2}}{2} I_{3}+\frac{\sqrt{a}}{2} I_{4} \\
& +\frac{b}{8}\left[\frac{3\left(I_{1}^{2}+I_{3}^{2}\right)}{4(a+2)}+\frac{3 I_{2}^{2}}{8(a+4)}+\frac{3 I_{4}^{2}}{8 a}\right. \\
& +\frac{3 I_{2}\left(I_{1}+I_{3}\right)}{\sqrt{2(a+2)(a+4)}}+\frac{3 I_{4}\left(I_{1}+I_{3}\right)}{\sqrt{2 a(a+2)}} \\
& \left.+\frac{3 I_{2} I_{4}}{\sqrt{2 a(a+4)}}\right],
\end{aligned}
$$

and the verification of the validity of the Kolmogorov's condition is straightforward.

3.4. Five Particles. There is a close resemblance between the two cases of tree and five particles.

We transform the quadratic part of the Hamiltonian (4) $n=5$ using the phonons $(\bar{q}, \bar{p})$ and scale in the usual way. Further, we use the notations $\Omega_{j}$ for short. Recall that

$$
\begin{aligned}
& \Omega_{1}=\Omega_{4}=\sqrt{a+\frac{(5-\sqrt{5})}{2}}, \\
& \Omega_{2}=\Omega_{3}=\sqrt{a+\frac{(5+\sqrt{5})}{2}}, \\
& \Omega_{5}=\sqrt{a} ;
\end{aligned}
$$

that is, $\Omega$ contains two internal resonances. The generators of the normal form in $(z, w)$ variables are

$$
z_{j} w_{j}, j=1, \ldots, 5 \text {, and } z_{1} w_{4}, z_{4} w_{1}, z_{2} w_{3}, z_{3} w_{2} .
$$

Then the truncated up to order 4 normal form $\bar{H}=H_{2}+\bar{H}_{4}$ for (4) $n=5$ reads

$$
\begin{aligned}
\bar{H} & =\frac{\Omega_{1}}{2}\left(z_{1} w_{1}+z_{4} w_{4}\right)+\frac{\Omega_{2}}{2}\left(z_{2} w_{2}+z_{3} w_{3}\right)+\frac{\Omega_{5}}{2} \\
& \cdot z_{5} w_{5}+\frac{b}{10}\left[\frac{9}{16 \Omega_{1}^{2}}\left(z_{1} w_{1}+z_{4} w_{4}\right)^{2}\right. \\
& +\frac{9}{16 \Omega_{2}^{2}}\left(z_{2} w_{2}+z_{3} w_{3}\right)^{2}+\frac{3}{8 \Omega_{5}^{2}}\left(z_{5} w_{5}\right)^{2} \\
& +\frac{3}{16 \Omega_{1}^{2}}\left(z_{1} w_{4}-z_{4} w_{1}\right)^{2}+\frac{3}{16 \Omega_{2}^{2}}\left(z_{2} w_{3}-z_{3} w_{2}\right)^{2} \\
& +\frac{3}{2 \Omega_{1} \Omega_{2}}\left(z_{1} w_{1}+z_{4} w_{4}\right)\left(z_{2} w_{2}+z_{3} w_{3}\right) \\
& +\frac{3}{2 \Omega_{1} \Omega_{5}}\left(z_{1} w_{1}+z_{4} w_{4}\right) z_{5} w_{5} \\
& \left.+\frac{3}{2 \Omega_{2} \Omega_{5}}\left(z_{2} w_{2}+z_{3} w_{3}\right) z_{5} w_{5}\right]
\end{aligned}
$$

or, written in the coordinates $(\bar{q}, \bar{p})$, it is

$$
\begin{aligned}
\bar{H} & =\frac{\Omega_{1}}{2}\left(\bar{p}_{1}^{2}+\bar{q}_{1}^{2}+\bar{p}_{4}^{2}+\bar{q}_{4}^{2}\right)+\frac{\Omega_{2}}{2}\left(\bar{p}_{2}^{2}+\bar{q}_{2}^{2}+\bar{p}_{3}^{2}\right. \\
& \left.+\bar{q}_{3}^{2}\right)+\frac{\Omega_{5}}{2}\left(\bar{p}_{5}^{2}+\bar{q}_{5}^{2}\right) \\
& +\frac{b}{10}\left[\frac{9}{16 \Omega_{1}^{2}}\left(\bar{p}_{1}^{2}+\bar{q}_{1}^{2}+\bar{p}_{4}^{2}+\bar{q}_{4}^{2}\right)^{2}\right. \\
& +\frac{9}{16 \Omega_{2}^{2}}\left(\bar{p}_{2}^{2}+\bar{q}_{2}^{2}+\bar{p}_{3}^{2}+\bar{q}_{3}^{2}\right)^{2}+\frac{3}{8 \Omega_{5}^{2}}\left(\bar{p}_{5}^{2}+\bar{q}_{5}^{2}\right)^{2} \\
& -\frac{3}{4 \Omega_{1}^{2}}\left(\bar{p}_{1} \bar{q}_{4}-\bar{q}_{1} \bar{p}_{4}\right)^{2}-\frac{3}{4 \Omega_{2}^{2}}\left(\bar{p}_{2} \bar{q}_{3}-\bar{q}_{2} \bar{p}_{3}\right)^{2} \\
& +\frac{3}{2 \Omega_{1} \Omega_{2}}\left(\bar{p}_{1}^{2}+\bar{q}_{1}^{2}+\bar{p}_{4}^{2}+\bar{q}_{4}^{2}\right)\left(\bar{p}_{2}^{2}+\bar{q}_{2}^{2}+\bar{p}_{3}^{2}+\bar{q}_{3}^{2}\right) \\
& +\frac{3}{2 \Omega_{1} \Omega_{5}}\left(\bar{p}_{1}^{2}+\bar{q}_{1}^{2}+\bar{p}_{4}^{2}+\bar{q}_{4}^{2}\right)\left(\bar{p}_{5}^{2}+\bar{q}_{5}^{2}\right) \\
& \left.+\frac{3}{2 \Omega_{2} \Omega_{5}}\left(\bar{p}_{2}^{2}+\bar{q}_{2}^{2}+\bar{p}_{3}^{2}+\bar{q}_{3}^{2}\right)\left(\bar{p}_{5}^{2}+\bar{q}_{5}^{2}\right)\right] .
\end{aligned}
$$

As it is seen, the above normal form is integrable with the following first integrals:

$$
\begin{aligned}
F_{1} & :=\bar{p}_{1}^{2}+\bar{q}_{1}^{2}+\bar{p}_{4}^{2}+\bar{q}_{4}^{2}, \\
F_{2} & :=\bar{p}_{2}^{2}+\bar{q}_{2}^{2}+\bar{p}_{3}^{2}+\bar{q}_{3}^{2}, \\
G_{1} & :=\bar{p}_{1} \bar{q}_{4}-\bar{q}_{1} \bar{p}_{4}, \\
G_{2} & :=\bar{p}_{2} \bar{q}_{3}-\bar{q}_{2} \bar{p}_{3}, \\
I_{5} & :=\bar{p}_{5}^{2}+\bar{q}_{5}^{2} .
\end{aligned}
$$

In a similar way as in [4] and above treated case of tree particles, in the domain where the first integrals are independent, we can introduce action-angle variables

$$
(\bar{q}, \bar{p}) \longrightarrow\left(F_{1}, F_{2}, G_{1}, G_{2}, I_{5}, \phi_{1}, \phi_{2}, \psi_{1}, \psi_{2}, \varphi_{5}\right)
$$

where $F_{j}, G_{j}, \quad j=1,2$ are as above and

$$
\begin{aligned}
\phi_{j}:= & \frac{1}{2} \arg \left(-\bar{p}_{5-j}-\bar{q}_{j}, \bar{p}_{j}-\bar{q}_{5-j}\right) \\
& +\frac{1}{2} \arg \left(\bar{p}_{5-j}-\bar{q}_{j}, \bar{p}_{j}+\bar{q}_{5-j}\right), \quad j=1,2, \\
\psi_{j}:= & \frac{1}{2} \arg \left(-\bar{p}_{5-j}-\bar{q}_{j}, \bar{p}_{j}-\bar{q}_{5-j}\right) \\
& -\frac{1}{2} \arg \left(\bar{p}_{5-j}-\bar{q}_{j}, \bar{p}_{j}+\bar{q}_{5-j}\right), \quad j=1,2, \\
\varphi_{5}:= & \arctan \frac{\bar{p}_{5}}{\bar{q}_{5}} .
\end{aligned}
$$

As above one can verify that $\left(F_{1}, F_{2}, G_{1}, G_{2}, I_{5}, \phi_{1}, \phi_{2}\right.$, $\left.\psi_{1}, \psi_{2}, \varphi_{5}\right)$ are canonical coordinates. Then the truncated up 
to order 4 normal form as a function of the action variables is

$$
\begin{aligned}
\bar{H} & =\frac{\Omega_{1}}{2} F_{1}+\frac{\Omega_{2}}{2} F_{2}+\frac{\Omega_{5}}{2} I_{5}+\frac{b}{10}\left[\frac{9}{16 \Omega_{1}^{2}}\left(F_{1}\right)^{2}\right. \\
& +\frac{9}{16 \Omega_{2}^{2}}\left(F_{2}\right)^{2}+\frac{3}{8 \Omega_{5}^{2}}\left(I_{5}\right)^{2}-\frac{3}{4 \Omega_{1}^{2}}\left(G_{1}\right)^{2} \\
& -\frac{3}{4 \Omega_{2}^{2}}\left(G_{2}\right)^{2}+\frac{3}{2 \Omega_{1} \Omega_{2}} F_{1} F_{2}+\frac{3}{2 \Omega_{1} \Omega_{5}} F_{1} I_{5} \\
& \left.+\frac{3}{2 \Omega_{2} \Omega_{5}} F_{2} I_{5}\right] .
\end{aligned}
$$

It is straightforward to check up whether the Kolmogorov's condition is valid.

Remark 6. Now, let us study the normal forms for the exceptional cases, listed in the end of Section 2. Surprisingly, the resonant terms corresponding to the resonant relations (21), (22), and (24) are missing in $H_{4}$. Solely the resonant terms corresponding to the resonant relations (23) appear in $\mathrm{H}_{4}$ in the following way:

$$
\frac{15 \sqrt{2}}{8 \Omega_{1} \sqrt{\Omega_{2} \Omega_{5}}}\left(z_{1}^{2} w_{2} w_{5}+w_{1}^{2} z_{2} z_{5}-w_{4}^{2} z_{2} z_{5}-z_{4}^{2} w_{2} w_{5}\right) .
$$

Therefore, the truncated normal form $\bar{H}=H_{2}+\bar{H}_{4}$ for (4) $n=5$ and $a=a_{3}$ is

$$
\begin{aligned}
\bar{H} & =\frac{\Omega_{1}}{2}\left(\bar{p}_{1}^{2}+\bar{q}_{1}^{2}+\bar{p}_{4}^{2}+\bar{q}_{4}^{2}\right)+\frac{\Omega_{2}}{2}\left(\bar{p}_{2}^{2}+\bar{q}_{2}^{2}+\bar{p}_{3}^{2}\right. \\
& \left.+\bar{q}_{3}^{2}\right)+\frac{\Omega_{5}}{2}\left(\bar{p}_{5}^{2}+\bar{q}_{5}^{2}\right)+\frac{b}{10}\left[\frac { 9 } { 1 6 \Omega _ { 1 } ^ { 2 } } \left(\bar{p}_{1}^{2}+\bar{q}_{1}^{2}+\bar{p}_{4}^{2}\right.\right. \\
& \left.+\bar{q}_{4}^{2}\right)^{2}+\frac{9}{16 \Omega_{2}^{2}}\left(\bar{p}_{2}^{2}+\bar{q}_{2}^{2}+\bar{p}_{3}^{2}+\bar{q}_{3}^{2}\right)^{2}+\frac{3}{8 \Omega_{5}^{2}}\left(\bar{p}_{5}^{2}\right. \\
& \left.+\bar{q}_{5}^{2}\right)^{2}-\frac{3}{4 \Omega_{1}^{2}}\left(\bar{p}_{1} \bar{q}_{4}-\bar{q}_{1} \bar{p}_{4}\right)^{2}-\frac{3}{4 \Omega_{2}^{2}}\left(\bar{p}_{2} \bar{q}_{3}\right. \\
& \left.-\bar{q}_{2} \bar{p}_{3}\right)^{2}+\frac{3}{2 \Omega_{1} \Omega_{2}}\left(\bar{p}_{1}^{2}+\bar{q}_{1}^{2}+\bar{p}_{4}^{2}+\bar{q}_{4}^{2}\right)\left(\bar{p}_{2}^{2}+\bar{q}_{2}^{2}\right. \\
& \left.+\bar{p}_{3}^{2}+\bar{q}_{3}^{2}\right)+\frac{3}{2 \Omega_{1} \Omega_{5}}\left(\bar{p}_{1}^{2}+\bar{q}_{1}^{2}+\bar{p}_{4}^{2}+\bar{q}_{4}^{2}\right)\left(\bar{p}_{5}^{2}\right. \\
& \left.+\bar{q}_{5}^{2}\right)+\frac{3}{2 \Omega_{2} \Omega_{5}}\left(\bar{p}_{2}^{2}+\bar{q}_{2}^{2}+\bar{p}_{3}^{2}+\bar{q}_{3}^{2}\right)\left(\bar{p}_{5}^{2}+\bar{q}_{5}^{2}\right) \\
& +\frac{15 \sqrt{2}}{4 \Omega_{1} \sqrt{\Omega_{2} \Omega_{5}}}\left[2\left(q_{2} p_{5}+p_{2} q_{5}\right)\left(q_{1} p_{1}-q_{4} p_{4}\right)\right. \\
& \left.\left.+\left(q_{2} q_{5}-p_{2} p_{5}\right)\left(q_{1}^{2}-p_{1}^{2}+p_{4}^{2}-q_{4}^{2}\right)\right]\right]
\end{aligned}
$$

It can be easily checked that the expressions (51) are no longer first integrals for this normal form. The known integrals are $\mathrm{H}_{2}$ and $\overline{\mathrm{H}}$ and that makes the integrability of this particular resonant normal form unclear.
3.5. Six Particles. In the diagonalized Hamiltonian system (4) $n=6$ we scale

$$
\begin{gathered}
\bar{q}_{1,5} \longrightarrow \frac{1}{\sqrt[4]{a+1}} \bar{q}_{1,5}, \\
\bar{p}_{1,5} \longrightarrow \sqrt[4]{a+1} \bar{p}_{1,5}, \\
\bar{q}_{2,4} \longrightarrow \frac{1}{\sqrt[4]{a+3}} \bar{q}_{2,4}, \\
\bar{p}_{2,4} \longrightarrow \sqrt[4]{a+3} \bar{p}_{2,4}, \\
\bar{q}_{3} \longrightarrow \frac{1}{\sqrt[4]{a+4}} \bar{q}_{3}, \\
\bar{p}_{3} \longrightarrow \sqrt[4]{a+4} \bar{p}_{3}, \\
\bar{q}_{6} \longrightarrow \frac{1}{\sqrt[4]{a}} \bar{q}_{6}, \\
\bar{p}_{6} \longrightarrow \sqrt[4]{a} \bar{p}_{6} .
\end{gathered}
$$

Recall that $\Omega_{1}=\Omega_{5}=\sqrt{a+1}, \Omega_{2}=\Omega_{4}=\sqrt{a+3}, \Omega_{3}=$ $\sqrt{a+4}, \Omega_{6}=\sqrt{a}$; that is, in these cases we again have two independent $1: 1$ resonances. In the variables $\left(z_{j}, w_{j}\right), j=$ $1, \ldots, 6$, the generators of the normal form are

$$
z_{j} w_{j} \text { and } z_{1} w_{5}, w_{1} z_{5}, z_{2} w_{4}, z_{4} w_{2}
$$

We normalize and find that the normal form $\bar{H}=H_{2}+\bar{H}_{4}$ up to order 4 is

$$
\begin{aligned}
& \bar{H}=\frac{\Omega_{1}}{2}\left(z_{1} w_{1}+z_{5} w_{5}\right)+\frac{\Omega_{2}}{2}\left(z_{2} w_{2}+z_{4} w_{4}\right)+\frac{\Omega_{3}}{2} \\
& \cdot z_{3} w_{3}+\frac{\Omega_{6}}{2} z_{6} w_{6}+\frac{b}{72}\left[\frac{27}{8 \Omega_{1}^{2}}\left(z_{1} w_{1}+z_{5} w_{5}\right)^{2}\right. \\
& +\frac{27}{8 \Omega_{2}^{2}}\left(z_{2} w_{2}+z_{4} w_{4}\right)^{2}+\frac{9}{4 \Omega_{3}^{2}}\left(z_{3} w_{3}\right)^{2} \\
& +\frac{9}{4 \Omega_{6}^{2}}\left(z_{6} w_{6}\right)^{2}+\frac{9\left(z_{3} w_{3}\right)\left(z_{6} w_{6}\right)}{\Omega_{3} \Omega_{6}}+\frac{9 z_{3} w_{3}}{\Omega_{1} \Omega_{3}}\left(z_{1} w_{1}\right. \\
& \left.+z_{5} w_{5}\right)+\frac{9 z_{3} w_{3}}{\Omega_{2} \Omega_{3}}\left(z_{2} w_{2}+z_{4} w_{4}\right)+\frac{9 z_{6} w_{6}}{\Omega_{1} \Omega_{6}}\left(z_{1} w_{1}\right. \\
& \left.+z_{5} w_{5}\right)+\frac{9 z_{6} w_{6}}{\Omega_{2} \Omega_{6}}\left(z_{2} w_{2}+z_{4} w_{4}\right) \\
& +\frac{9}{8}\left(\frac{z_{1} w_{5}-w_{1} z_{5}}{\Omega_{1}}-\frac{z_{2} w_{4}-w_{2} z_{4}}{\Omega_{2}}\right)^{2} \\
& +\frac{9}{2 \Omega_{1} \Omega_{2}}\left(z_{1} w_{1} z_{4} w_{4}+z_{2} w_{2} z_{5} w_{5}+3 z_{1} w_{1} z_{2} w_{2}\right. \\
& +3 z_{4} w_{4} z_{5} w_{5}-\left(z_{1} w_{5}+w_{1} z_{5}\right)\left(z_{2} w_{4}+w_{2} z_{4}\right) \\
& \left.\left.+\frac{1}{2}\left(z_{1} w_{5}-w_{1} z_{5}\right)\left(z_{2} w_{4}-w_{2} z_{4}\right)\right)\right] \text {. }
\end{aligned}
$$


Before returning to the variables $(\bar{q}, \bar{p})$ we denote

$$
\begin{aligned}
& F_{1}:=\bar{p}_{1}^{2}+\bar{q}_{1}^{2}+\bar{p}_{5}^{2}+\bar{q}_{5}^{2}, \\
& F_{2}:=\bar{p}_{2}^{2}+\bar{q}_{2}^{2}+\bar{p}_{4}^{2}+\bar{q}_{4}^{2}, \\
& I_{3}:=\bar{p}_{3}^{2}+\bar{q}_{3}^{2}, \\
& I_{6}:=\bar{p}_{6}^{2}+\bar{q}_{6}^{2} .
\end{aligned}
$$

Then the truncated normal form $\bar{H}=H_{2}+\bar{H}_{4}$ becomes

$$
\begin{aligned}
\bar{H} & =\frac{\Omega_{1}}{2} F_{1}+\frac{\Omega_{2}}{2} F_{2}+\frac{\Omega_{3}}{2} I_{3}+\frac{\Omega_{6}}{2} I_{6}+\frac{b}{8}\left[\frac{3 F_{1}^{2}}{8 \Omega_{1}^{2}}\right. \\
& +\frac{3 F_{2}^{2}}{8 \Omega_{2}^{2}}+\frac{I_{3}^{2}}{4 \Omega_{3}^{2}}+\frac{I_{6}^{2}}{4 \Omega_{6}^{2}}+\frac{F_{1} F_{2}}{2 \Omega_{1} \Omega_{2}}+\frac{I_{3} I_{6}}{\Omega_{3} \Omega_{6}}+\frac{I_{3} F_{1}}{\Omega_{1} \Omega_{3}} \\
& +\frac{I_{3} F_{2}}{\Omega_{2} \Omega_{3}}+\frac{I_{6} F_{1}}{\Omega_{1} \Omega_{6}}+\frac{I_{6} F_{2}}{\Omega_{2} \Omega_{6}}-\frac{1}{2}\left(\frac{\bar{p}_{1} \bar{q}_{5}-\bar{q}_{1} \bar{p}_{5}}{\Omega_{1}}\right. \\
& \left.-\frac{\bar{p}_{2} \bar{q}_{4}-\bar{q}_{2} \bar{p}_{4}}{\Omega_{3}}\right)^{2}+\frac{1}{\Omega_{1} \Omega_{2}}\left(\left(\bar{p}_{1}^{2}+\bar{q}_{1}^{2}\right)\left(\bar{p}_{2}^{2}+\bar{q}_{2}^{2}\right)\right. \\
& +\left(\bar{p}_{4}^{2}+\bar{q}_{4}^{2}\right)\left(\bar{p}_{5}^{2}+\bar{q}_{5}^{2}\right) \\
& -2\left(\bar{q}_{1} \bar{q}_{5}+\bar{p}_{1} \bar{p}_{5}\right)\left(\bar{q}_{2} \bar{q}_{4}+\bar{p}_{2} \bar{p}_{4}\right) \\
& \left.\left.-\left(\bar{p}_{1} \bar{q}_{5}-\bar{q}_{1} \bar{p}_{5}\right)\left(\bar{p}_{2} \bar{q}_{4}-\bar{q}_{2} \bar{p}_{4}\right)\right)\right] .
\end{aligned}
$$

This normal form is integrable: the independent first integrals are

$$
\begin{aligned}
& F_{1}, F_{2}, I_{3}, I_{6}, G:=\left(\bar{p}_{1} \bar{q}_{5}-\bar{q}_{1} \bar{p}_{5}\right)-\left(\bar{p}_{2} \bar{q}_{4}-\bar{q}_{2} \bar{p}_{4}\right) \text { and } \\
& \bar{H}_{4} .
\end{aligned}
$$

However, the construction of all action-angle variables is unclear so far.

This finishes the proof of Theorem 3.

\section{Concluding Remarks}

This paper presents partial results on integrability of normal forms of the periodic KG lattices.

We study the normal forms because the original systems are nonintegrable. This is proven rigorously in the case of two degrees of freedom (Theorem 2) and that is one of the differences with the FPU chain. One can carry out the nonintegrability proof for $n=3$ in the same line, but with more efforts. There is a technical difficulty to carry through that proof in the higher-dimensions, however. The variational equation (VE) does not split nicely and one needs either a tool to deal with higher dimensional (NVE) or another particular solution with the VE along it suitable enough. Nevertheless, we claim that the periodic KG lattice is nonintegrable for all $n \geq 2$.

The calculation of the normal forms goes in the standard way, because we treat only low dimensions. It is also facilitated by the assumption an $a$. The result in Theorem 3 allows us in most cases to view the periodic KG Hamiltonian (4) as a perturbation of a nondegenerate Liouville integrable Hamiltonian, namely, the truncated up to order four BirkhoffGustavson normal form. One can also verify the other KAM condition, known as Arnold-Moser's condition [6].

As a consequence we have found an integrable KAM nondegenerate normal form of $1: 2: 2$ Hamiltonian resonance (see Remark 5).

The outcome of Theorem 3 suggests that when one considers the integrability of the normal forms in higherdimensional periodic KG lattices, certain finite set of values of $a$ even in the domain (3) has to be ruled out initially. For the values of this set there probably exist additional fourthorder resonant relations, the corresponding normal forms are probably more complicated than the generic ones, and, hence, more difficult to study.

In any case, the results of this paper serve to understand the lattices with many particles. We intend to study them with the approach of Rink [4], based on using the symmetry properties, which we also enjoy here, to construct suitable normal forms.

Finally, we do not address here the symmetric invariant manifolds in the KG lattices because they can be retrieved from [14].

\section{Appendix}

\section{Nonintegrability of Periodic KG Lattice with $n=2$}

In this Appendix we give the proof of Theorem 2. It is based on Ziglin-Morales-Ruiz-Ramis theory. The main result of this theory merely says that if a Hamiltonian system is completely integrable, then the identity component of the Galois group of the variational equation along certain particular solution is abelian.

In the applications if one finds out that the identity component of the Galois group is noncommutative, then this implies nonintegrability. However, if this component turns out to be abelian, one needs additional steps to prove nonintegrability as it is carried out below.

The necessary facts and results about differential Galois theory and its relations with the integrability of Hamiltonian systems, enough for our purposes, are written succinctly in Section 2 in [15] and we do not repeat them here. We refer the reader to [16-19] for a more detailed exposition.

The proof goes in the following lines. We obtain a particular solution and write the variational equation along this solution. It appears that the identity component of its differential Galois group is abelian. In order to obtain an obstacle to the integrability, we study the higher variational equations. Their differential Galois groups are in principle solvable. One possible way to show that some of them is not abelian is to find a logarithmic term in the corresponding solution (see $[17,18])$. We obtain such a logarithmic term in the solution of the second variational equation when $b \neq 0$. Then the nonintegrability of the Hamiltonian system follows. 
Proof. Suppose $b \neq 0$. First we bring the quadratic part into diagonal form. For this purpose we perform a symplectic change of variables in (5) $q=Y \bar{q}, p=Y \bar{p}, q=\left(q_{1}, q_{2}\right), p=$ $\left(p_{1}, p_{2}\right)$ with $Y=(1 / \sqrt{2})\left(\begin{array}{cc}1 & 1 \\ -1 & 1\end{array}\right)$ (see Section 2$)$. In the new coordinates the Hamiltonian reads (we skip the bars for simplicity here)

$$
\begin{aligned}
H= & \frac{1}{2}\left(p_{1}^{2}+p_{2}^{2}\right)+\frac{1}{2}\left[(4+a) q_{1}^{2}+a q_{2}^{2}\right] \\
& +\frac{b}{4}\left(q_{1}^{4}+6 q_{1}^{2} q_{2}^{2}+q_{2}^{4}\right) .
\end{aligned}
$$

Remark A.1. The Hamiltonian (A.1) is of the form

$$
\begin{aligned}
H= & \frac{1}{2}\left(p_{1}^{2}+p_{2}^{2}\right)+\frac{1}{2}\left[\alpha_{1} q_{1}^{2}+\alpha_{2} q_{2}^{2}\right] \\
& +\frac{b}{4}\left(q_{1}^{4}+6 q_{1}^{2} q_{2}^{2}+q_{2}^{4}\right),
\end{aligned}
$$

which is known to be integrable when $\alpha_{1}=\alpha_{2}$ (see, e.g., [20] and the references therein). However, this is not the case here. We did not succeed in finding a nonintegrability proof for the case $\alpha_{1} \neq \alpha_{2}$; that is why we present it here.

It is also assumed that throughout this Appendix all variables are complex: $t \in \mathbb{C}, q_{j} \in \mathbb{C}, p_{j} \in \mathbb{C}, j=1,2$. The following proposition is immediate.

Proposition A.2. The Hamiltonian system corresponding to (A.1) admits a particular solution

$$
\begin{aligned}
& q_{1}^{0}(t)=\operatorname{sn}\left(\sqrt{a+4+\frac{b}{2}} t, k\right), \\
& p_{1}^{0}(t)=\frac{d}{d t} q_{1}^{0}(t), \\
& q_{2}^{0}(t)=p_{2}^{0}(t)=0,
\end{aligned}
$$

where sn is the Jacobi elliptic function with the module $k=$ $\sqrt{(-b / 2) /(4+a+b / 2)}$.

It is straightforward that $T_{1}=4 K / \sqrt{a+4+b / 2}$ and $T_{2}=2 i K^{\prime} / \sqrt{a+4+b / 2}$ are the periods of (A.3). Here $K$, $K^{\prime}$ are the complete elliptic integrals of the first kind. In the parallelogram of the periods, the solution (A.3) has two simple poles

$$
\begin{aligned}
& t_{1}=\frac{i K^{\prime}}{\sqrt{a+4+b / 2}}, \\
& t_{2}=\frac{2 K+i K^{\prime}}{\sqrt{a+4+b / 2}} .
\end{aligned}
$$

Denoting by $\xi_{j}^{(1)}=d q_{j}, \eta_{j}^{(1)}=d p_{j}, j=1,2$, the variational equations (VE) (written as second-order equations) are

$$
\begin{aligned}
& \ddot{\xi}_{1}^{(1)}=-\left[(4+a)+3 b\left(q_{1}^{0}(t)\right)^{2}\right] \xi_{1}^{(1)}, \\
& \ddot{\xi}_{2}^{(1)}=-\left[a+3 b\left(q_{1}^{0}(t)\right)^{2}\right] \xi_{2}^{(1)} .
\end{aligned}
$$

Since $d H=p_{1}^{0}(t) \eta_{1}^{(1)}+(4+a) q_{1}^{0}(t) \xi_{1}^{(1)}+b\left(q_{1}^{0}(t)\right)^{3} \xi_{1}^{(1)}$ does not depend on $\left(\xi_{2}^{(1)}, \eta_{2}^{(1)}\right)$, then (A.6) stands for normal variational equation (NVE); to be more specific:

$$
\ddot{\xi}_{2}^{(1)}+\left[a+3 b \operatorname{sn}^{2}\left(\sqrt{4+a+\frac{b}{2}} t, k\right)\right] \xi_{2}^{(1)}=0 .
$$

This equation has regular singularities at $t_{1,2}$; that is, it is a Fuchsian one.

From the expansion of the sn in the neighborhood of the pole $t_{1}$, we have

$$
\begin{aligned}
& q_{1}^{0}(t)=\frac{1}{\sqrt{-b / 2}}\left[\frac{1}{t-t_{1}}+\frac{4+a}{6}\left(t-t_{1}\right)+c\left(t-t_{1}\right)^{3}\right. \\
& \left.\quad+d\left(t-t_{1}\right)^{5}+\cdots\right],
\end{aligned}
$$

where $c$ is an arbitrary constant and

$$
d=\frac{1}{14}\left[\frac{16}{27}+4 c+\frac{4 a}{9}+a c+\frac{a^{2}}{9}+\frac{a^{3}}{108}\right] .
$$

It is not difficult to see from (A.7) and (A.8) that the indicial equation [21] at $t=t_{1}$

$$
r(r-1)-6=0
$$

has roots $r_{1,2}=-2,3$ (compare with the expansions below). Therefore, the monodromy around $t_{1}$ is trivial and can not serve as an obstacle to integrability $[22,23]$ (similarly for the monodromy around $t_{2}$ ).

In fact, we can say more about the identity component of the Galois group of (VE) (A.5), (A.6). Notice that each of the variational equations (VE) is a Lamé equation in Jacobi form.

Proposition A.3. The identity component of the differential Galois group of (VE) (A.5), (A.6) is abelian.

Proof. The analysis is facilitated by the fact that (VE) is split into two second-order differential equations with the coefficients in the field of elliptic functions.

Let us start with the first equation (A.5) (the consideration of the second equation is similar). It is straightforward that one solution of (A.5) is $\xi_{1,1}^{(1)}=\dot{q}_{1}^{0}(t)$. This solution belongs to the field of the coefficients. The other linearly independent solution is

$$
\xi_{1,2}^{(1)}=\xi_{1,1}^{(1)} \int \frac{d t}{\left(\xi_{1,1}^{(1)}\right)^{2}},
$$

which does not belong in general to the coefficient field. Then the identity component of its Galois group is isomorphic to $\left(\begin{array}{ll}1 & 0 \\ \nu_{1} & 1\end{array}\right)$. Therefore, the identity component of the Galois group of $(\mathrm{VE})$ is isomorphic to

$$
\left(\begin{array}{cccc}
1 & 0 & 0 & 0 \\
v_{1} & 1 & 0 & 0 \\
0 & 0 & 1 & 0 \\
0 & 0 & v_{2} & 1
\end{array}\right), \quad \nu_{1}, \nu_{2} \in \mathbb{C}
$$

and it is clearly abelian. 
In what follows we need the expansions around $t_{1}$ of the fundamental systems of solutions with unit Wronskians for (A.5) and (A.6). For the expansions of (A.5) we get

$$
\begin{aligned}
\xi_{1,1}^{(1)}= & \frac{1}{\left(t-t_{1}\right)^{2}}-\frac{4+a}{6}-3 c\left(t-t_{1}\right)^{2} \\
& -\left(\frac{8+6 a}{27}+2 c+\frac{a c}{2}+\frac{a^{2}}{18}+\frac{a^{3}}{216}\right)\left(t-t_{1}\right)^{4} \\
& +\cdots, \\
\xi_{1,2}^{(1)}= & \frac{1}{5}\left(t-t_{1}\right)^{3}+\cdots
\end{aligned}
$$

and similarly for (A.5)

$$
\begin{aligned}
\xi_{2,1}^{(1)} & =\frac{1}{\left(t-t_{1}\right)^{2}}-\frac{8+a}{6}-\frac{a+6-9 c}{3}\left(t-t_{1}\right)^{2} \\
& +\left(\frac{56+11 a}{27}-\frac{91 c+9 a c}{18}-\frac{a^{2}}{54}-\frac{a^{3}}{216}\right) \\
\cdot & \left(t-t_{1}\right)^{4}+\cdots, \\
\xi_{2,2}^{(1)} & =\frac{1}{5}\left(t-t_{1}\right)^{3}+\cdots .
\end{aligned}
$$

One can see that these expansions are in fact convergent since $t_{1}$ is a regular singular point (cf. [21]). Hence, the fundamental matrix $X(t)$ of $(\mathrm{VE})$ is

$$
\begin{aligned}
X(t) & =\left(\begin{array}{cccc}
\xi_{1,1}^{(1)} & \xi_{1,2}^{(1)} & 0 & 0 \\
\dot{\xi}_{1,1}^{(1)} & \dot{\xi}_{1,2}^{(1)} & 0 & 0 \\
0 & 0 & \xi_{2,1}^{(1)} & \xi_{2,2}^{(1)} \\
0 & 0 & \dot{\xi}_{2,1}^{(1)} & \dot{\xi}_{2,2}^{(1)}
\end{array}\right), \\
X^{-1}(t) & =\left(\begin{array}{cccc}
\dot{\xi}_{1,2}^{(1)} & -\xi_{1,2}^{(1)} & 0 & 0 \\
-\dot{\xi}_{1,1}^{(1)} & \xi_{1,1}^{(1)} & 0 & 0 \\
0 & 0 & \dot{\xi}_{2,2}^{(1)} & -\xi_{2,2}^{(1)} \\
0 & 0 & -\dot{\xi}_{2,1}^{(1)} & \xi_{2,1}^{(1)}
\end{array}\right) .
\end{aligned}
$$

Now, let us consider the higher variational equations along the particular solution (A.3). We put

$$
\begin{aligned}
& q_{1}=q_{1}^{0}(t)+\varepsilon \xi_{1}^{(1)}+\varepsilon^{2} \xi_{1}^{(2)}+\varepsilon^{3} \xi_{1}^{(3)}+\cdots, \\
& p_{1}=\dot{q}_{1} \\
& q_{2}=0+\varepsilon \xi_{2}^{(1)}+\varepsilon^{2} \xi_{2}^{(2)}+\varepsilon^{3} \xi_{2}^{(3)}+\cdots \\
& p_{2}=\dot{q}_{2}
\end{aligned}
$$

where $\varepsilon$ is a formal parameter and substitute these expressions into the Hamiltonian system governed by (A.1). Comparing the terms with the same order in $\varepsilon$ we obtain consequently the variational equations up to any order.
The first variational equation is, of course, (A.5), (A.6). For the second variational equation we have

$$
\begin{aligned}
& \dot{\xi}_{1}^{(2)}=\eta_{1}^{(2)}, \\
& \dot{\eta}_{1}^{(2)}=-\left[4+a+3 b\left(q_{1}^{0}(t)\right)^{2}\right] \xi_{1}^{(2)}+K_{1}, \\
& \dot{\xi}_{2}^{(2)}=\eta_{2}^{(2)}, \\
& \dot{\eta}_{2}^{(2)}=-\left[a+3 b\left(q_{1}^{0}(t)\right)^{2}\right] \xi_{2}^{(2)}+K_{2},
\end{aligned}
$$

where

$$
\begin{aligned}
& K_{1}=-3 b q_{1}^{0}(t)\left[\left(\xi_{1}^{(1)}\right)^{2}+\left(\xi_{2}^{(1)}\right)^{2}\right] \\
& K_{2}=-6 b q_{1}^{0}(t) \xi_{1}^{(1)} \xi_{2}^{(1)} .
\end{aligned}
$$

In this way we can obtain a chain of linear nonhomogeneous differential equations

$$
\begin{aligned}
& \dot{\xi}^{(k)}=A(t) \xi^{(k)}+f_{k}\left(\xi^{(1)}, \ldots, \xi^{(k-1)}\right), \\
& \\
& k=1,2, \ldots,
\end{aligned}
$$

where $A(t)$ is the linear part of the Hamiltonian equations along the particular solution and $f_{1}=0$. The above equation is called $k$ th variational equation $\left(\mathrm{VE}_{k}\right)$. If $X(t)$ is a fundamental matrix of $\left(\mathrm{VE}_{1}\right)$, then the solutions of $\left(\mathrm{VE}_{k}\right), k>1$, can be found by

$$
\xi^{(k)}=X(t) c(t),
$$

where

$$
\dot{c}=X^{-1}(t) f_{k} .
$$

Let us study the local solutions of $\left(\mathrm{VE}_{2}\right)$. In our case $f_{2}=$ $\left(0, K_{1}, 0, K_{2}\right)^{T}$ and from (A.15) we get

$$
X^{-1}(t) f_{2}=\left(-\xi_{1,2}^{(1)} K_{1}, \xi_{1,1}^{(1)} K_{1},-\xi_{2,2}^{(1)} K_{2}, K_{2}\right)^{T}
$$

We are looking for a component of $X^{-1}(t) f_{2}$ with a nonzero residuum at $t=t_{1}$. This would imply the appearance of a logarithmic term. Indeed, the residue at $t=t_{1}$ of $\xi_{2,1}^{(1)} K_{2}$ with the specific representatives is

$$
\begin{aligned}
& \operatorname{Res}_{t=t_{1}}\left(-6 b \xi_{2,1}^{(1)} q_{1}^{0}(t) \xi_{1,1}^{(1)} \xi_{2,1}^{(1)}\right) \\
& \quad=\frac{6 b}{\sqrt{-b / 2}}\left[\frac{a^{3}}{252}+\frac{a^{2}}{21}+\frac{4 a}{21}+\frac{3 a c}{7}+\frac{73 c+16}{63}\right] .
\end{aligned}
$$

Since $c$ is an arbitrary parameter, we choose it in such a way that the expression in the square brackets does not vanish for $a>0$. There are many such values of $c$, say $c=1$. Recall that by assumption $b \neq 0$, we have obtained a nonzero residuum at $t=t_{1}$, which implies the appearance of a logarithmic term in the solutions of $\left(\mathrm{VE}_{2}\right)$. Then its Galois group is solvable but not abelian. Hence, we conclude the nonintegrability of the Hamiltonian system (5). 


\section{Conflicts of Interest}

The author declares that they have no conflicts of interest.

\section{Acknowledgments}

This work is supported by Bulgarian Fund "Scientific Research" (Grant DN 02-5).

\section{References}

[1] A. M. Morgante, M. Johansson, G. Kopidakis, and S. Aubry, "Standing wave instabilities in a chain of nonlinear coupled oscillators," Physica D: Nonlinear Phenomena, vol. 162, no. 1-2, pp. 53-94, 2002.

[2] H. H. de Jong, Quasiperiodic breathers in systems of weakly coupled pendulums [Ph. D. Thesis], University of Groningen, Groningen, Netherlands, 1999.

[3] B. Rink and F. Verhulst, "Near-integrability of periodic FPUchains," Physica A: Statistical Mechanics and its Applications, vol. 285, no. 3, pp. 467-482, 2000.

[4] B. Rink, "Symmetry and resonance in periodic FPU chains," Communications in Mathematical Physics, vol. 218, no. 3, pp. 665-685, 2001.

[5] O. Christov, "Near Integrability in Low Dimensional GrossNeveu Models," Zeitschrift für Naturforschung A, vol. 66, no. 8-9, 2011.

[6] V. I. Arnold, Mathematical Methods of Classical Mechanics, Springer, 1978.

[7] R. Abraham and J. Marsden, Foundations of Mechanics, Benjamin, Reading, Mass, USA, 1987.

[8] V. I. Arnold, V. V. Kozlov, and A. I. Neishtadt, "Mathematical aspects of classical and celestial mechanics," in Dynamical systems III, Springer, New York, NY, USA, 2006.

[9] A. N. Kolmogorov, "On conservation of conditionally periodic motions for a small change in Hamilton's function," Doklady Akademii Nauk SSSR, vol. 98, no. 4, pp. 527-530, 1954 (Russian).

[10] V. Arnold, "Proof of a theorem of A. N. Kolmogorov on the invariance of quasi-periodic motions under small perturbations of the Hamiltonian," Uspekhi Matematicheskikh Nauk, vol. 18, no. 5, pp. 13-40, 1963 (Russian).

[11] J. Moser, "Convergent series expansions for quasi-periodic motions," Mathematische Annalen, vol. 169, pp. 136-176, 1967.

[12] F. Verhulst, "Symmetry and integrability in hamiltonian normal forms," in Symmetry and Perturbation Theory, D. Bambusi and G. Gaeta, Eds., Farenze, 1998.

[13] F. Verhulst, "Integrability and non-integrability of Hamiltonian normal forms," Acta Applicandae Mathematicae, vol. 137, pp. 253-272, 2015.

[14] B. Rink, "Symmetric invariant manifolds in the Fermi-PastaUlam lattice," Physica D: Nonlinear Phenomena, vol. 175, no. 1-2, pp. 31-42, 2003.

[15] O. Christov and G. Georgiev, "On the integrability of a system describing the stationary solutions in Bose-Fermi mixtures," Chaos, Solitons \& Fractals, vol. 77, pp. 138-148, 2015.

[16] J. Morales-Ruiz, "Differential galois theory and non integrability of Hamiltonian systems," in Progress in Mathematics, vol. 179, Birkhäuser, 1999.

[17] J. J. Morales-Ruiz, J.-P. Ramis, and C. Simó, "Integrability of Hamiltonian systems and differential Galois groups of higher variational equations," Annales Scientifiques de l'Ecole Normale Superieure, vol. 40, no. 6, pp. 845-884, 2007.

[18] J. J. Morales-Ruiz and J.-P. Ramis, "Integrability of dynamical systems through differential Galois theory: a practical guide," in Differential Algebra, Complex Analysis and Orthogonal Polynomials, P. Acosta-Humanez and F. Marcellan, Eds., vol. 509 of Contemporary Mathematics, 2010.

[19] M. Singer and M. van der Put, "Galois theory of linear differential equations," in Grundlehren der Mathematischen Wissenschaften, vol. 328, Springer, 2003.

[20] S. a. Kasperczuk, "A note on the integrability of Hamiltonian systems," Letters in Mathematical Physics, vol. 61, no. 3, pp. 199204, 2002.

[21] E. Wittaker and G. Watson, A Course of Modern Analysis, Cambridge University Press, Cambridge, UK, 1927.

[22] S. L. Ziglin, "Branching of solutions and nonexistence of first integrals in Hamiltonian mechanics. I," Functional Analysis and Its Applications, vol. 16, no. 3, pp. 181-189, 1982.

[23] S. L. Ziglin, "Branching of solutions and the nonexistence of first integrals in Hamiltonian mechanics. II," Functional Analysis and Its Applications, vol. 17, no. 1, pp. 6-17, 1983. 


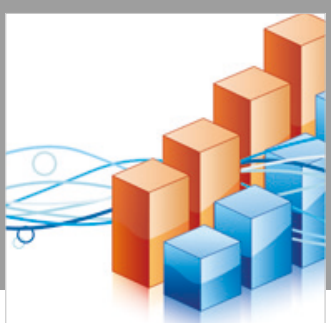

Advances in

Operations Research

\section{-n-m}
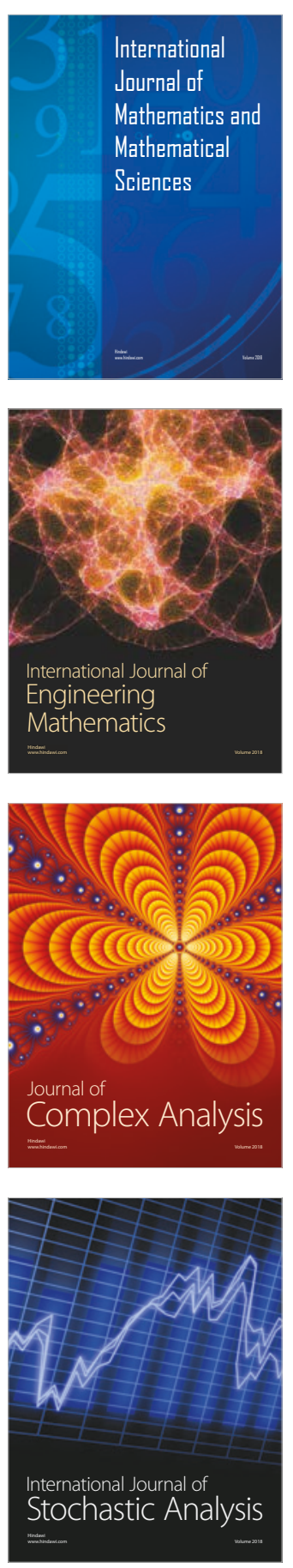
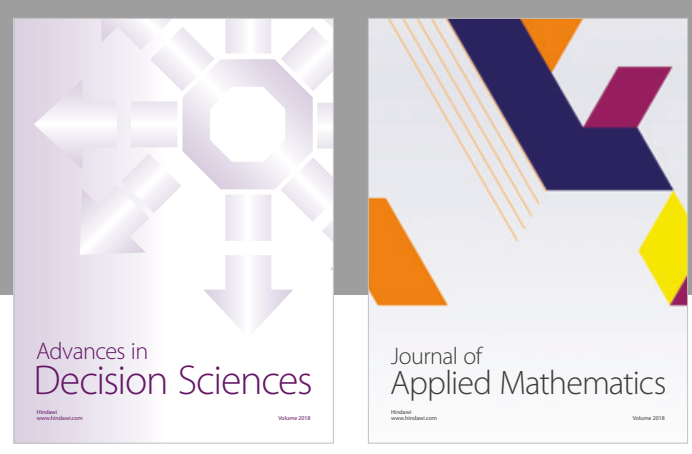

Journal of

Applied Mathematics
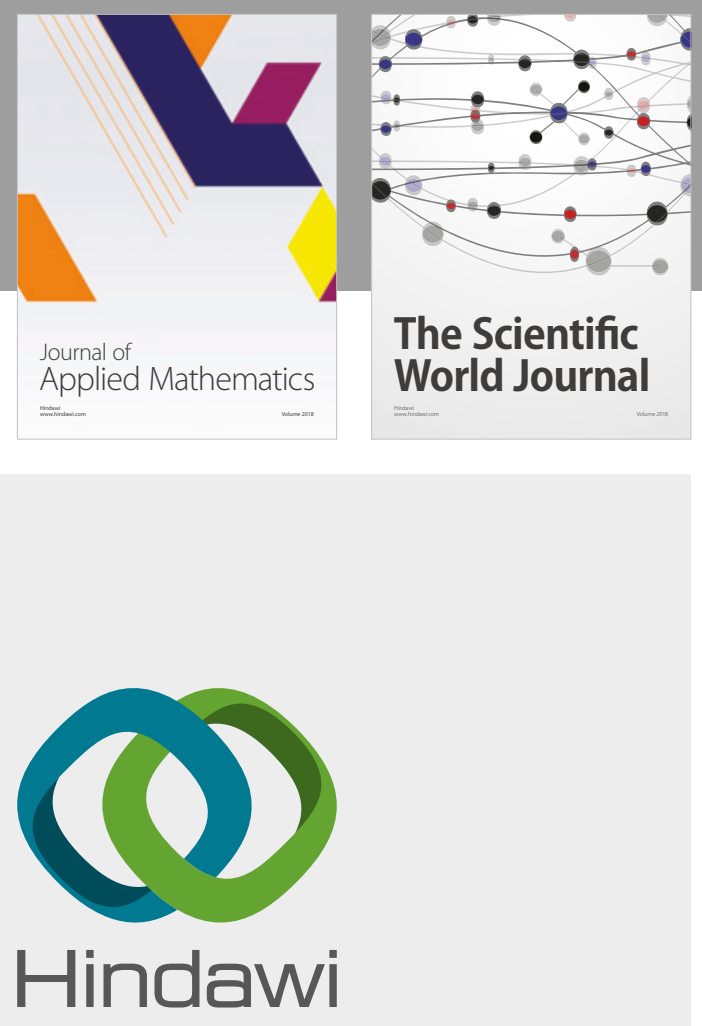

Submit your manuscripts at

www.hindawi.com

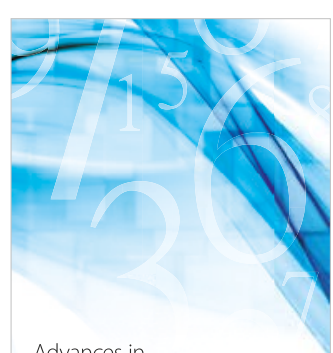

Advances in
Numerical Analysis
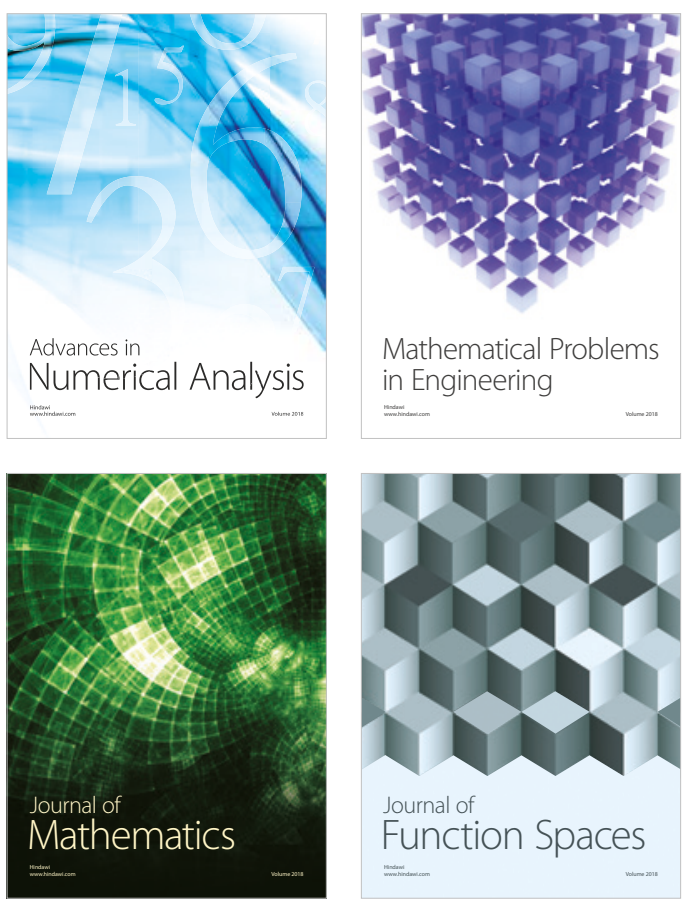

Mathematical Problems in Engineering

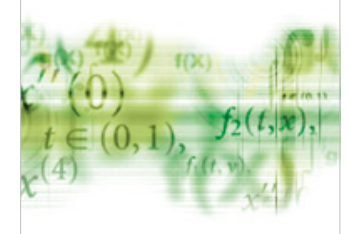

International Journal of

Differential Equations

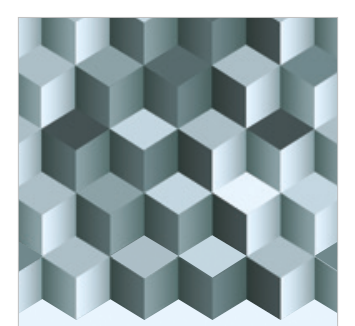

Journal of

Function Spaces

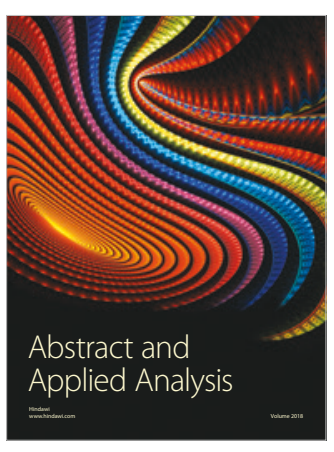

The Scientific

World Journal

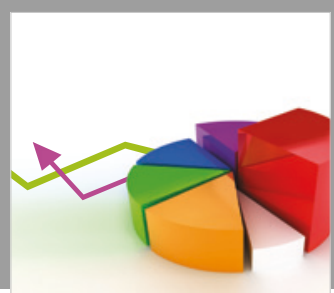

Journal of

Probability and Statistics
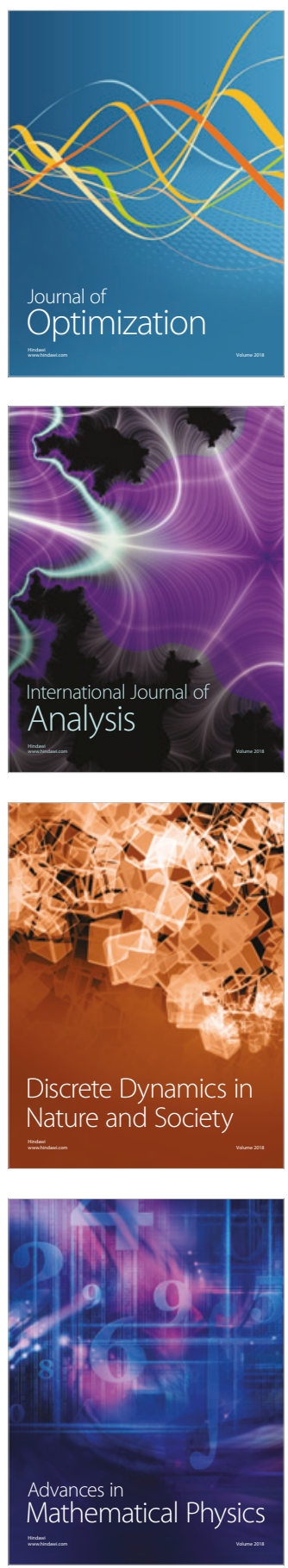